\title{
High-Fidelity Measurement of Qubits Encoded in Multilevel Superconducting Circuits
}

\author{
Salvatore S. Elder $\oplus^{*},+\dagger$ Christopher S. Wang, ${ }^{\dagger \dagger \dagger}$ Philip Reinhold, Connor T. Hann, \\ Kevin S. Chou, ${ }^{\S}$ Brian J. Lester, ${ }^{\|}$Serge Rosenblum, ${ }^{\natural}$ Luigi Frunzio, Liang Jiang, ${ }^{* *}$ and Robert J. Schoelkopf ${ }^{\ddagger}$ \\ Department of Applied Physics and Physics, Yale University, New Haven, Connecticut 06520, USA \\ and Yale Quantum Institute, Yale University, New Haven, Connecticut 06520, USA
}

(Received 5 August 2019; revised manuscript received 15 October 2019; published 2 January 2020; corrected 19 June 2020)

\begin{abstract}
Qubit measurements are central to quantum information processing. In the field of superconducting qubits, standard readout techniques are limited not only by the signal-to-noise ratio, but also by state relaxation during the measurement. In this work, we demonstrate that the limitation due to relaxation can be suppressed by using the many-level Hilbert space of superconducting circuits: In a multilevel encoding, the measurement is corrupted only when multiple errors occur. Employing this technique, we show that we can directly resolve transmon gate errors at the level of one part in $10^{3}$. Extending this idea, we apply the same principles to the measurement of a logical qubit encoded in a bosonic mode and detected with a transmon ancilla, implementing a proposal by Hann et al. [Phys. Rev. A 98, 022305 (2018)]. Qubit state assignments are made based on a sequence of repeated readouts, further reducing the overall infidelity. This approach is quite general, and several encodings are studied; the codewords are more distinguishable when the distance between them is increased with respect to photon loss. The trade-off between multiple readouts and state relaxation is explored and shown to be consistent with the photon-loss model. We report a logical assignment infidelity of $5.8 \times 10^{-5}$ for a Fock-based encoding and $4.2 \times 10^{-3}$ for a quantum error correction code (the $S=2, N=1$ binomial code). Our results not only improve the fidelity of quantum information applications, but also enable more precise characterization of process or gate errors.
\end{abstract}

DOI: 10.1103/PhysRevX.10.011001

Subject Areas: Quantum Physics, Quantum Information

\section{INTRODUCTION}

Quantum information processing (QIP) involves many tasks. One requirement, crucial to any QIP experiment, is the ability to measure a qubit or qubit register. Given a superposition $|\psi\rangle=\alpha\left|0_{L}\right\rangle+\beta\left|1_{L}\right\rangle$, our task is to collapse the state and detect the corresponding classical bit of information. That is, we should extract a "0" with probability $|\alpha|^{2}$ and a "1" with probability $|\beta|^{2}$, projecting onto

\footnotetext{
*sal.elder@yale.edu

†christopher.wang@yale.edu

" robert.schoelkopf@yale.edu

${ }^{\S}$ Present address: Quantum Circuits, Inc., New Haven, Connecticut 06520, USA.

"Present address: Atom Computing, Berkeley, California 94710, USA.

"Present address: Department of Condensed Matter Physics, Weizmann Institute of Science, Rehovot, Israel.

*** Present address: Pritzker School of Molecular Engineering, University of Chicago, Chicago, Illinois 60637, USA.

${ }^{\dagger}$ These authors contributed equally to this work.

Published by the American Physical Society under the terms of the Creative Commons Attribution 4.0 International license. Further distribution of this work must maintain attribution to the author(s) and the published article's title, journal citation, and DOI.
}

the state $\left|0_{L}\right\rangle$ or $\left|1_{L}\right\rangle$, respectively. A canonical example of qubit measurement is at the end of a quantum computation, after which the experimenter measures the qubit array and infers a useful result. Experimental implementations of quantum computing requirements, including feedbackbased state preparation [1], gate calibration, and errorsyndrome extraction for quantum error correction (QEC) [2-6], also rely on qubit measurements. Outside of quantum computing applications, measurement is used in quantum key distribution [7], enhanced sensing [8], teleportation of states or gates [9-11], and fundamental tests of locality and entanglement $[12,13]$. Although the particular metrics depend on the application at hand, better measurements translate into better results in all of the examples mentioned. Furthermore, improvements in qubit measurement improve the calibration and characterization [14] of gates and other quantum operations. Finally, we point out that, in order to implement large quantum circuits, measurements must improve alongside advances in gate quality and the number of qubits. For example, for fixed singlequbit measurement error, the probability that an entire register of qubits will be measured correctly decreases exponentially in the size of the register.

Much progress has been made in the experimental implementation of single-shot qubit measurements in a variety of systems. Single-shot measurements of qubits 
based on trapped ions [15-17], electron spins in quantum dots [18-20], and superconducting circuits [21,22] have all been demonstrated with fidelities higher than $99 \%$. Improvements have been made in a variety of ways; interesting approaches include mapping a spin state onto a metastable charge state [18] and the repeated readout of an ion state using an ancilla [15].

In all of the systems mentioned above, state relaxation is a major limitation to the measurement fidelity. In a continuous measurement, some signal (such as a photomultiplier current or the phase of a microwave tone) is recorded and compared to the expected response corresponding to each qubit state. Because noise causes uncertainty in the assignment of individual outcomes, we would generally like to acquire a signal for a long time in order to improve the measurement contrast. On the other hand, $T_{1}$ events during the measurement, in which the qubit relaxes from its excited to ground state, lead to incorrect assignment of the initial state [23].

In this work, we overcome the first-order $T_{1}$ limit by encoding qubits in multilevel systems. If the physical states representing the 0 and 1 bit are separated by multiple energy levels, then a single relaxation event will not corrupt the 0 or 1 which is encoded. For this reason, qubit encodings with a larger distance between codewords with respect to the dominant error channel can be measured with much improved fidelity.

As a simple and direct example of our approach, in Sec. III, we study the measurement of a transmon qubit $(\hat{t})$ dispersively coupled [24] to a readout resonator $(\hat{r})$. In particular, using higher levels of the transmon is shown to improve the fidelity by 2 orders of magnitude in the device studied in our work. In Sec. IV, in order to more fully demonstrate the advantage of multilevel encodings, we encode a qubit in a harmonic oscillator implemented as a $\lambda / 4$ coaxial superconducting cavity. Using a bosonic mode allows us to systematically explore different qubit encodings, including Fock-based encodings as well as errorcorrectable binomial codes [25]. The information in this "storage" mode $(\hat{s})$ is read out using the dispersively coupled transmon as an ancilla according to a recent proposal [26]. In this proposal, the storage-ancilla interaction is used to map information from the storage onto the ancilla, and the ancilla-readout interaction is used to read out the ancilla state.

\section{BACKGROUND}

Before going further, it is worth clarifying what is meant by encoding and measuring a bit in a multilevel system. Traditionally, a qubit is identified with a two-level system such as a spin- $\frac{1}{2}$ particle in a magnetic field. In this case, there is no trouble identifying the two eigenstates with the values of a bit: $|\uparrow\rangle$ represents a 0 , say, and $|\downarrow\rangle$ represents a 1 . To measure the bit encoded by such a system means performing a projective measurement of an eigenstate of (a)
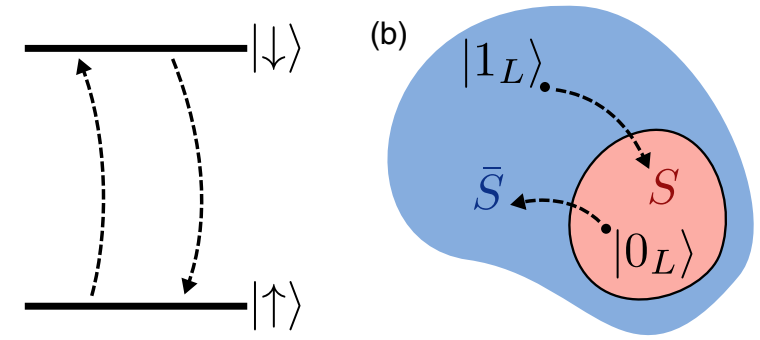

FIG. 1. Cartoon illustrating qubit readout errors. (a) In a twolevel system, qubit readout means determining the energy eigenstate of the system at time $t=0$. Dashed arrows indicate incorrect assignments $P(\uparrow|| \downarrow\rangle)$ and $P(\downarrow \| \uparrow\rangle)$, which may be due to detector noise or state transitions. (b) In a many-dimensional Hilbert space, reading out a single bit requires partitioning the Hilbert space into two sets containing the encoded 0 and 1 states. Instead of an energy eigenstate, membership in $S$ or its complement $\bar{S}=\mathcal{H} \backslash S$ is measured. The dashed arrows now indicate the assignment errors $\left.P\left(S|| 1_{L}\right\rangle\right)$ and $\left.P\left(\bar{S}|| 0_{L}\right\rangle\right)$.

the system. Finally, to incorrectly measure the bit encoded by such a system means that state transitions or detector noise cause the experimenter to record a state initially $|\downarrow\rangle$ as "0" or vice versa. These simple ideas are sketched in Fig. 1(a), where dashed arrows indicate incorrect measurements.

Actual implementations of qubits, however, may contain more than two energy levels. In this case, the definition of a qubit is a matter of convention. For example, the transmon $[27,28]$ is an anharmonic oscillator with several energy levels $(|g\rangle,|e\rangle,|f\rangle,|h\rangle, \ldots)$ in which the ground and first excited states are often operated as a qubit. This situation is possible because the $|g\rangle-|e\rangle$ transition is detuned by many linewidths from all other transitions, so that it can be individually addressed by a single rf drive. In this paper, we consider representing and measuring information encoded in the higher states.

Even more care is required when dealing with a harmonic oscillator, in which many transitions fall within the mode linewidth and there is no obvious notion of a qubit. In fact, several continuous-variable codes [25,29-35] have been studied, in which two states in Hilbert space, $\left|0_{L}\right\rangle$ and $\left|1_{L}\right\rangle$, are designated as the logically encoded 0 and 1 states, respectively. What does it mean to measure qubits encoded in this way? To answer this question, we imagine partitioning the Hilbert space $\mathcal{H}$ into two subsets, $S$ and $\bar{S}$, containing $\left|0_{L}\right\rangle$ and $\left|1_{L}\right\rangle$, respectively. An ideal measurement of the encoded bit is a projective measurement of the membership in $S$. The positive-operator-valued measure (POVM) operators [36] corresponding to such a measurement are

$$
\begin{gathered}
\mathcal{M}_{0}=\sum_{|\psi\rangle \in S}|\psi\rangle\langle\psi|, \\
\mathcal{M}_{1}=\mathbb{1}-\mathcal{M}_{0} .
\end{gathered}
$$


(a)

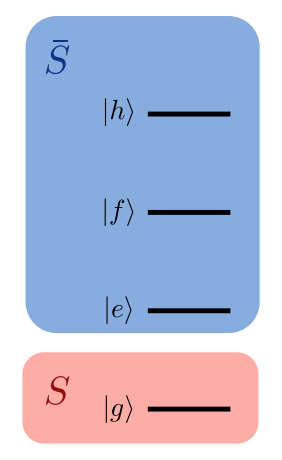

(b)

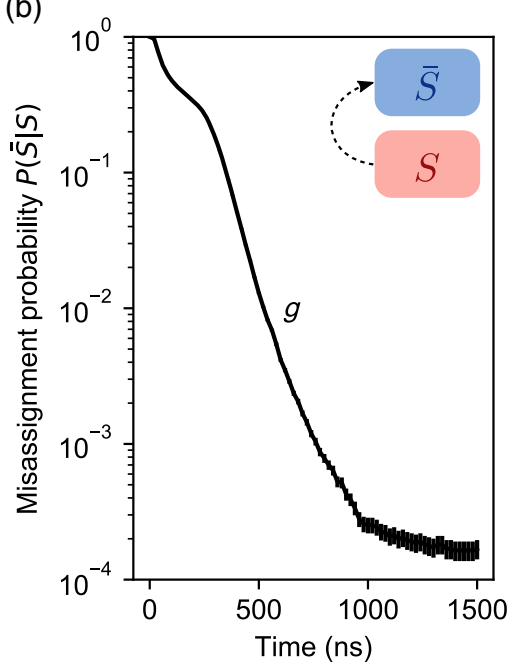

(c)

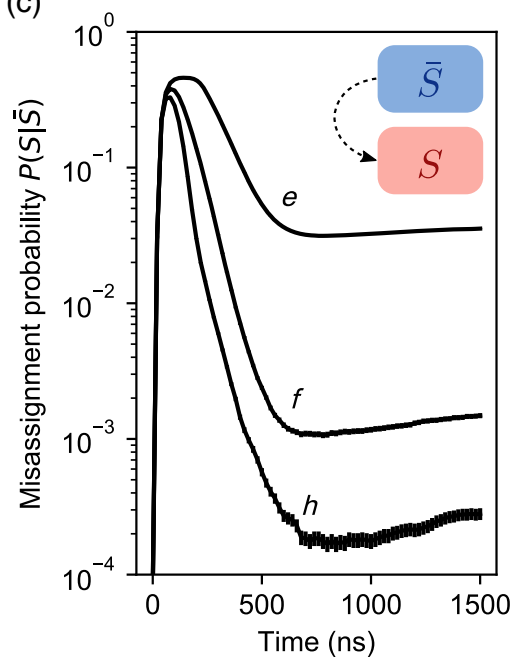

FIG. 2. High-fidelity measurement of a transmon. (a) Representation of the measurement performed. The first four states of the transmon are individually prepared, measured, and classified as belonging to $S$ or $\bar{S}$ based on the measurement record. (b) The probability that the ground state $|g\rangle$ is incorrectly assigned as belonging to $\bar{S}$, plotted as a function of the length of the measurement signal used to make the classification. The initial shape of the curve is related to the ring-up time of the readout resonator. The misassignment probability decreases as a function of time, because collecting more signal improves the separation of readout signals. However, the improvement stops once the misassignment probability is comparable to the probability that the transmon has gained a photon. Collecting more signal causes additional misassignments. (c) The probability that an excited state $|e\rangle,|f\rangle$, or $|h\rangle$ is incorrectly assigned as belonging to $S$. Again, after some initial transient behavior related to an edge case in the classification method, the misassignment probability improves as the signal is collected for a longer time. Eventually, relaxation events cause misassignments, and the curves increase for large acquisition times. The probability that all excitations are lost, causing erroneous assignment to $S$, is much smaller when a higher excited state is prepared. The exact misassignment probabilities achieved depend on many factors, especially the relaxation time $T_{1}^{(g e)} \approx 51 \mu$ s and thermal population $\bar{n}_{t} \approx 0.4 \%$.

Finally, the fidelity of such a measurement is defined [23] as

$$
\left.\left.F=1-P\left(S|| 1_{L}\right\rangle\right)-P\left(\bar{S}|| 0_{L}\right\rangle\right),
$$

where $P(a \mid s)$ is the probability that an assignment $a$ will be made, given that the true initial state is $s$. The fidelity offers a simple, unified metric with which we can compare the measurement of different codes.

\section{MULTILEVEL TRANSMON MEASUREMENT}

In the standard approach to superconducting qubit measurement, a readout resonator is dispersively coupled to a transmon, meaning that the frequency of the resonator mode is shifted if the transmon is in an excited state. By driving the resonator and recording the amplitude and phase of the response, one can therefore infer the transmon state. Of course, there is inevitably noise on top of the average resonator response. Integrating the signal for a longer time provides more separation between the two averages. However, transmons undergo random transitions between eigenstates with a characteristic time $T_{1}$. After a certain fraction of $T_{1}$, the measurement signal no longer provides useful information about the initial state; therefore, the signal-to-noise ratio competes with the lifetime of the information being measured [23].
Suppose that, instead of asking whether the qubit was initially in its ground state $|g\rangle$ or first excited state $|e\rangle$, we ask a slightly different question: Was the qubit initially in its ground state $|g\rangle$ or in an excited state $|e\rangle,|f\rangle,|h\rangle, \ldots$ ? Extending the logic above, the $n$th excited state remains distinguishable from $|g\rangle$ until $n$ relaxation events occur. Therefore, we are able to acquire the signal for a longer time, leading to a better signal-to-noise ratio. Additionally, the figure of merit $t_{m} / T_{1} \ll 1$ for relaxation to the ground state becomes $\left(t_{m} / T_{1}\right)^{n}$ when discriminating between $|g\rangle$ and the $n$th excited state, where $t_{m}$ is the measurement acquisition time [37]. This result enables us to perform high-fidelity measurements of transmon states. The measurement error for a related system was previously studied as a function of the signal-to-noise ratio and the number of excited states [39].

In order to test or characterize a high-fidelity transmon measurement, we first need to prepare the states $|g\rangle,|e\rangle$, $|f\rangle$, and $|h\rangle$ accurately. This preparation is done by applying the appropriate pulse sequence, then performing a preliminary "check" measurement, and postselecting on measurements which give us a high confidence that the correct state has been prepared. Because we record all outcomes following a successful state preparation, this method is a fair way to characterize the measurement fidelity [40]. 
We test the fidelity by measuring the transmon and comparing the resulting assignment to the state which is prepared. The resulting misassignment probabilities are plotted in Fig. 2 as functions of the measurement time. To classify an individual measurement record $z(t)$ up to a particular time $t_{m}$, the record is compared to the average signals $\bar{z}_{g}(t), \ldots, \bar{z}_{h}(t)$ corresponding to the $|g\rangle, \ldots,|h\rangle$ states. The classifier outputs the state $s$ for which $\sum_{t=0}^{t_{m}}\left|z(t)-z_{s}(t)\right|^{2}$ is minimal. These labels are used to determine whether a particular realization is assigned correctly as either the ground state or an excited state.

We can understand the shapes of these plots qualitatively as follows: In the first part of the measurement, we acquire more signal, and the misassignment probabilities decrease. Eventually, however, we are limited by state relaxation, and the measurement no longer improves. If we continue to collect signal and classify the states naively, then we actually start to make more misassignments due to the state relaxation. The probability that state $|e\rangle,|f\rangle$, or $|h\rangle$ decays to $|g\rangle$ has $t^{1}, t^{2}$, or $t^{3}$ dependence, leading to the improvement in measurement fidelity shown in Fig. 2(c). We observe that if a transmon is prepared in the $|g\rangle-|h\rangle$ manifold, then it can be read out with a measurement infidelity of $P(S|| h\rangle)+P(\bar{S}|| g\rangle)=(4.0 \pm 0.5) \times 10^{-4}$.

We can apply these ideas to the more usual task of measuring " $g$ " or " $e$ " using a shelving technique. The idea is simple: We apply a rapid $e-f \pi$ pulse, $X_{\pi}^{e f}=$ $|f\rangle\langle e|+| e\rangle\langle f|$, to the transmon immediately before measuring it. Because the coherence of the $e-f$ manifold is comparable to that of the $g-e$ manifold, the shelving operation can be performed reliably. In this way, we transform the problem of distinguishing $|g\rangle$ from $|e\rangle$ into the problem of distinguishing $|g\rangle$ from $|f\rangle$, which can be done with higher fidelity. This transformation has been previously explored as a method of increasing the contrast of qubit measurements with a latching readout [42]. We observe that, in such a scheme, the misassignment of $|e\rangle$ as $g$ is a second-order error. On the other hand, the misassignment probability is directly sensitive to preparation error. Therefore, if $|e\rangle$ is prepared with a pulse, then the error in that pulse limits the performance of the shelved measurement.

However, this result means that a shelved measurement has a resolution comparable to the transmon gate errors. A measurement with such resolution is highly desirable in circuit QED, in which single-qubit gate errors are typically obscured by much larger measurement errors and must be characterized by indirect techniques $[43,44]$. We now show, using the above method for improved $|g\rangle-|e\rangle$ readout, how one can calibrate gates in a way that reduces state preparation and measurement (SPAM) errors. For example, a $g-e \pi$ pulse, $X_{\pi}^{g e}=|g\rangle\langle e|+| e\rangle\langle g|$, would typically be calibrated by the following procedure. (i) Prepare the transmon in its ground state $|g\rangle$. (ii) Apply the pulse with a variable amplitude. (iii) Measure the state of the qubit.
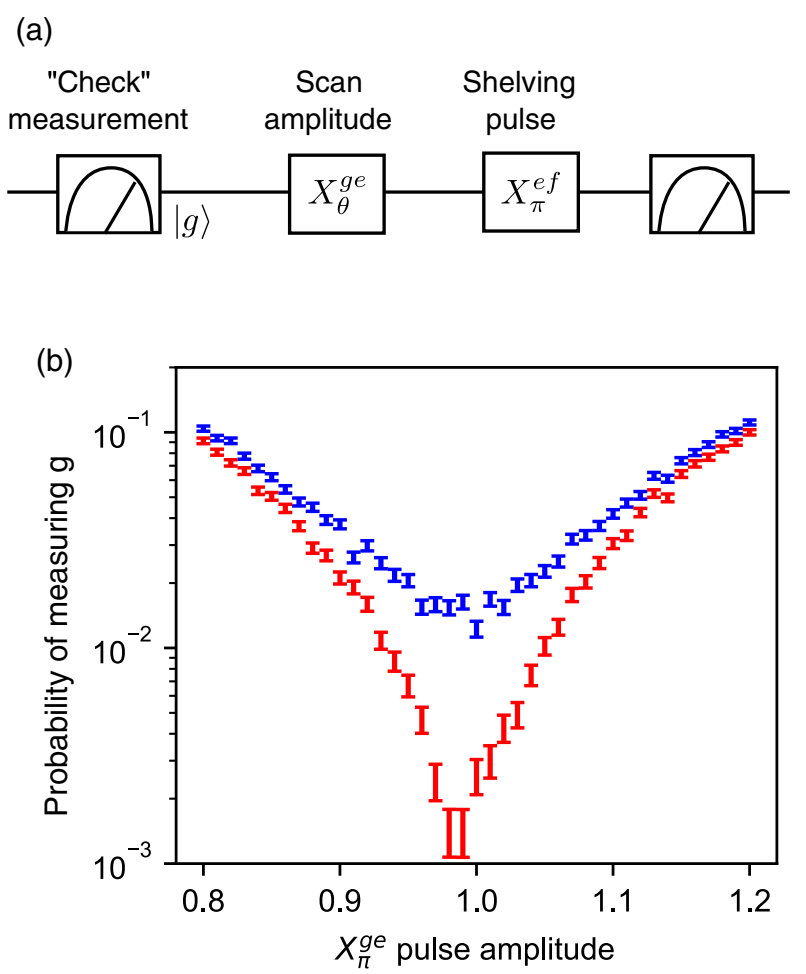

FIG. 3. Improved calibration and characterization of the gate error. (a) Schematic and (b) results of the shelved measurement demonstration. After the ground state is prepared, a $g-e \pi$ pulse is applied with a variable amplitude, and the transmon is measured. An $e-f$ "shelving" pulse before the measurement greatly improves the visibility of $g-e$ Rabi oscillation. The unshelved measurement is shown in blue, and the shelved measurement is shown in red.

The result of such a procedure is shown in Fig. 3 in blue. The minimum value of this curve, $(1.2 \pm 0.1) \times 10^{-2}$, is the inferred gate error, uncorrected for SPAM errors. Alternatively, the same procedure can be performed using shelving as described above. As shown in red in Fig. 3, the visibility is greatly improved because of the reduced measurement error. We therefore obtain, by direct measurement, an improved bound of $(1.4 \pm 0.3) \times 10^{-3}$ on the gate error.

We have shown above, how to characterize a gate to within a small factor. A Lindblad [45] master equation simulation including the effects of relaxation, dephasing, and leakage predicts a residual $g$ population of $P_{g}=3.7 \times$ $10^{-4}$ after the $g-e \pi$ pulse. This population is the quantity we would like to be able to measure directly. Similar calculations predict $P_{g}^{\text {shelved }}=7.3 \times 10^{-4}$ at the end of the $e-f$ pulse, which is somewhat larger due to additional relaxation during the $e-f$ pulse. Finally, in order to roughly estimate the probability of reading out $g$, we calculate $P_{g}^{\text {meas }}=9.1 \times 10^{-4}$ halfway through the measurement [41]. These calculations show that, although there is some error in the shelved measurement itself, it is comparable to the pulse error we wish to characterize. 
We stress that, if the gate fidelity $\left(\sim t_{\text {gate }} / T_{2}\right)$ were to improve, then the shelved measurement would improve with it.

\section{MEASUREMENT OF LOGICALLY ENCODED QUBITS}

As mentioned earlier, continuous-variable (CV) encodings of quantum information in bosonic modes offer a promising route to fault-tolerant quantum computation [29,31,46]. Instead of encoding a logical qubit in many physical qubits, $\mathrm{CV}$ schemes use an infinite-dimensional Hilbert space to build the redundancy needed for error correction into a single oscillator or storage mode. In this section, we show how redundancy and distance can be used to improve logical qubit measurements. We implement a proposal [26] to measure multiphoton encodings in a harmonic storage mode with high fidelity. The method consists of mapping the encoded information onto an ancilla, reading out the ancilla, resetting the ancilla, and repeating these steps several times.

To understand the advantage of a repeated readout scheme, first consider the error associated with mapping information onto the ancilla and reading it out. If this process is noisy, it is liable to give the wrong answer, but if it is quantum nondemolition with respect to the state of the storage mode - that is, if the readout does not induce extra transitions between eigenstates - then the storage mode can be read out repeatedly. To make an assignment of the bit encoded by the storage mode, one could take a majority vote of the outcomes obtained via the ancilla. In this way, many individual readouts of the encoded information can be combined to a single measurement with a greatly reduced probability of error. Next, consider the relaxation of the storage mode itself. As photons are lost incoherently from the storage, the information encoded by the storage is corrupted, limiting the ability of any measurement to extract the initially encoded bit. For example, if information is encoded in Fock states $|0\rangle$ and $|1\rangle$, then the relaxation rate $\kappa_{\downarrow}$ times the total mapping, readout, and reset time $\tau$ (with the appropriate prefactor) sets a lower bound on the probability of a measurement error. On the other hand, if the logical codewords are separated by $L$ Fock states, then the measurement is robust up to $L$ relaxation events. Therefore, the bound scales like $\left(\kappa_{\downarrow} \tau\right)^{L}$, an exponential improvement.

The measurement infidelity is estimated for various multiphoton encodings which are repeatedly read out using

(a)

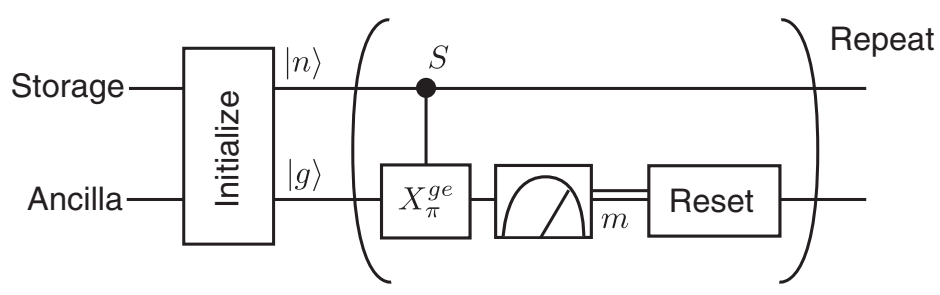

(b)

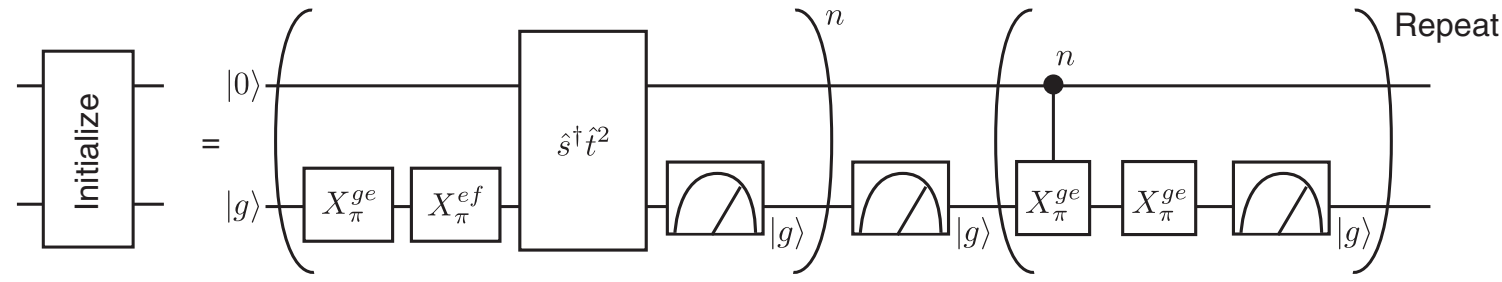

(c)

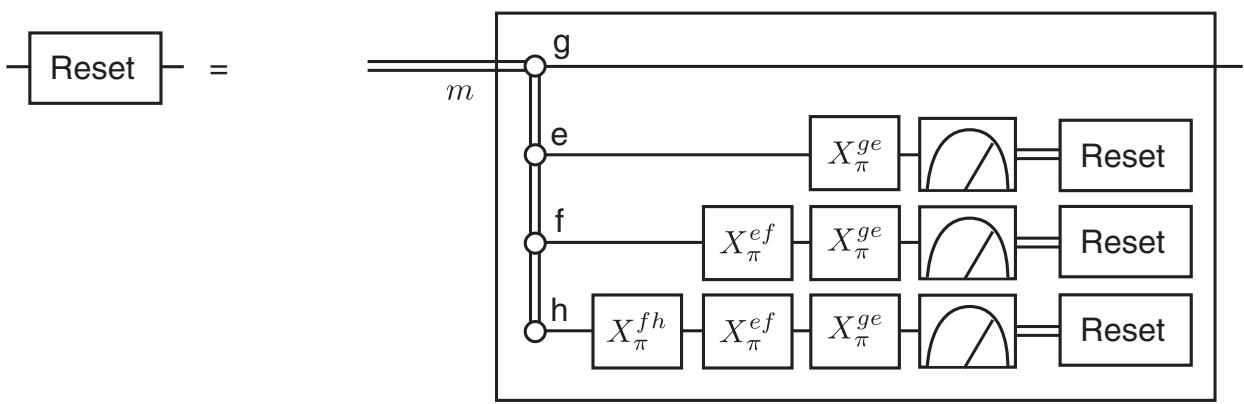

FIG. 4. Schematic of the repeated measurement procedure. (a) Block diagram showing how an encoded bit is measured. We first prepare one of several Fock states $|n\rangle$ in the storage mode. We then perform several readout cycles, each time obtaining an outcome. Each cycle consists of a decode pulse which excites the ancilla conditioned on the encoded bit, followed by a readout and reset of the ancilla. (b) The initialization procedure uses number-selective pulses and ancilla readouts to verify that the correct state has been prepared. This verification is crucial in order to demonstrate a sensitive detection. (c) Each reset consists of a real-time feedforward protocol, which ensures that the ancilla is in its ground state $|g\rangle$ at the end of each cycle. 
an ancilla as described above. It was shown [26] that the measurement infidelity can be broken into two contributions, corresponding to the observations above: first, the probability that a majority of individual readouts will give the wrong answer (ignoring state relaxation) and, second, the probability that the state will relax from $S$ into $\bar{S}$ (or vice versa) during the first half of the repeated readout sequence. These terms are given by

$$
\begin{aligned}
1-F= & L\left(\left\lceil\frac{N}{2}\right\rceil \kappa_{\downarrow} \tau\right)^{L-1}+\left(\left\lceil\frac{N}{2}\right\rceil \kappa_{\uparrow} \tau\right)^{2} \\
& +\left(\begin{array}{c}
N \\
\lceil N / 2\rceil
\end{array}\right) \delta_{0}^{\lceil N / 2\rceil}+\left(\begin{array}{c}
N \\
\lceil N / 2\rceil
\end{array}\right) \delta_{1}^{\lceil N / 2\rceil}
\end{aligned}
$$

for the family of Fock codes $\left|0_{L}\right\rangle=|0\rangle,\left|1_{L}\right\rangle=|L\rangle$. In the equation above, $N$ denotes the number of measurements made, $\kappa_{\downarrow(\uparrow)}$ is the rate of energy loss (gain) in the storage mode, and $\delta_{0(1)}$ is the probability of a readout error during a single round of measurement when the $\left|0_{L}\right\rangle\left(\left|1_{L}\right\rangle\right)$ state is prepared. We choose $S=\operatorname{span}\{|0\rangle,|1\rangle\}$ to measure this encoding so that the measurement of the $|0\rangle$ state is robust to the gain of a single photon.

The measurement protocol described allows us to perform many independent readouts of an encoded qubit using only a single ancilla. This procedure is accomplished using an adaptive feedforward scheme, which is illustrated in Fig. 4. A high-level description is given in Fig. 4(a), with further implementation details shown in Figs. 4(b) and 4(c). We next discuss the experimental implementation of the protocol.

Once the system is initialized, the first step of the measurement is to map the information encoded in the storage mode onto the state of the ancilla. This step is shown as an $S$-controlled $X_{\pi}^{g e}$ gate in Fig. 4(a). The realization of this entangling gate is based on the dispersive interaction between $\hat{s}$ and $\hat{t}$, which imparts an ancilla frequency shift which depends on the number of excitations in the storage mode:

$$
\begin{aligned}
\hat{H} / \hbar & =\omega_{t} \hat{t}^{\dagger} \hat{t}+\chi_{s t} \hat{s}^{\dagger} \hat{s}^{\dagger} \hat{t}+\cdots \\
& =\left(\omega_{t}+\chi_{s t} \hat{s}^{\dagger} \hat{s}\right) \hat{t}^{\dagger} \hat{t}+\cdots
\end{aligned}
$$

An ancilla pulse with spectral content near the frequencies corresponding to the $n$ in $S$ has the effect of flipping the ancilla state if and only if the storage state is in $S$. For example, when measuring the Fock codes, the ancilla is excited if the storage is in the state $|0\rangle$ or $|1\rangle$.

After the information encoded by the storage mode is mapped onto the ancilla, the state of the ancilla is read out. The outcome $m$ provides information about the encoded qubit state and provides the repeated measurement protocol with one vote. (a)

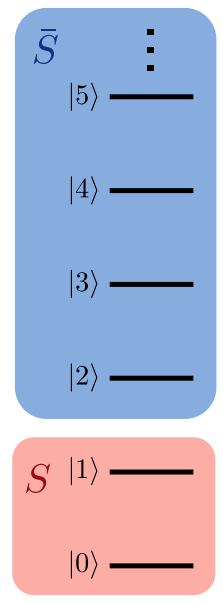

(b)

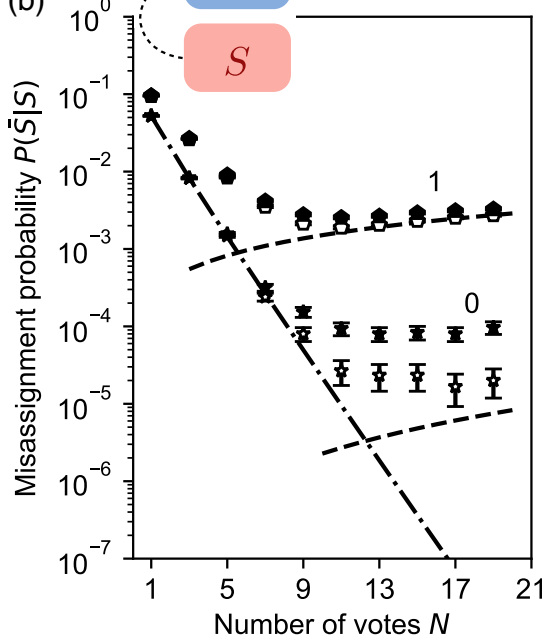

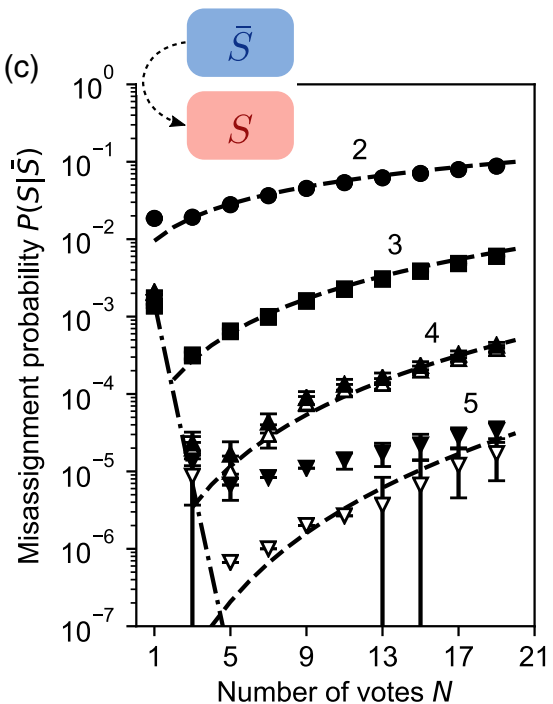

FIG. 5. Enhancement of measurement fidelity with the code distance. (a) In these experiments, we excite the ancilla if the storage mode has fewer than two photons in it. In doing so, we read out whether the storage state is in $S$ or $\bar{S}$ as shown. (b) Probability that, when a state in $S$ is prepared in the storage mode, it is assigned incorrectly as $\bar{S}$. The state $|0\rangle$ or $|1\rangle$ is prepared in the storage mode, as indicated by the labels on the plot. The data show that the fidelity improves exponentially with the number of votes, until excitation out of the $S$ subspace limits the measurement. Dash-dotted lines show the terms in the theoretical prediction corresponding to majority voting, dashed lines show terms corresponding to state transitions, and closed symbols show the experimental data. Open symbols indicate postselection on successful ancilla resets. (c) Probability that, when a state in $\bar{S}$ is prepared in the storage mode, it is assigned incorrectly as $S$. Again, we see that majority voting suppresses the misassignment probability but that for large $N$ the misassignment probability increases due to photon loss. States of higher photon number are measured with a smaller error, because the measurement is robust to more photon-loss events. 
However, the outcome of the readout also determines the operation which must be performed in order to reset the ancilla. The readout is calibrated to distinguish between the states $|g\rangle,|e\rangle,|f\rangle$, and $|h\rangle$. Ideally, the ancilla stays in the $|g\rangle-|e\rangle$ manifold, but resolving higher states enables a more robust reset operation. The reset protocol is shown in Fig. 4(c) as a recursive block diagram. It relies on real-time logic implemented on field-programmable gate array (FPGA) cards and does not terminate until it successfully records the ancilla in its ground state $|g\rangle$. Once the ancilla is reset to its ground state, it is available for additional measurements. In this way, the map-measure-reset cycle is repeated many times in order to extract a single highfidelity measurement of the logical qubit.

In order to demonstrate a high-fidelity measurement of the storage mode, it is crucial that the initialization step prepare states accurately. As shown in Fig. 4(b), number states are prepared by creating two excitations in the ancilla, then using a sideband interaction to convert them into a storage excitation [47], and repeating this process the desired number of times. This process is associated with significant initialization errors (on the order of several percent overall when preparing states of multiple photons), which would dominate over the measurement infidelity and prevent us from making any conclusions about the performance of our protocol. Therefore, the ancilla is read out after each application of the sideband interaction, to ensure that it returns to its ground state. Finally, after the state creation routine is finished, number-selective pulses and ancilla readouts are used to repeatedly check that the correct state was prepared. Only if all checks pass is the state preparation accepted.

The results of a repeated measurement experiment are plotted in Fig. 5 and illustrate all of the expected behavior. Figure 5(a) defines the operator which is measured; in this case, the goal is to measure whether the storage was initially in the state $|0\rangle$ or $|L\rangle$ for some $L \geq 2$. We plot the results of a majority vote as a function of the number of readouts taken. Furthermore, the assignment errors are split into two types: $\left|0_{L}\right\rangle$ incorrectly assigned as 1 and vice versa. As more readouts are incorporated into the majority vote, the measurement fidelity improves, because mapping and readout errors are suppressed. Eventually, however, this suppression competes with state transitions in the storage mode itself, causing the measurement to degrade once too many readouts are taken. We also observe that, as the distance between codewords $(L)$ increases, the measurement fidelity improves dramatically. These trends are captured by their theoretical description [26] and follow the predictions of Eq. (4), shown in dashed and dash-dotted lines, respectively.

The open symbols in Fig. 5 represent postselection of successful reset operations. By removing $0.2 \%$ of records corresponding to nonideal ancilla resets, we obtain much better agreement with the theoretical predictions. These

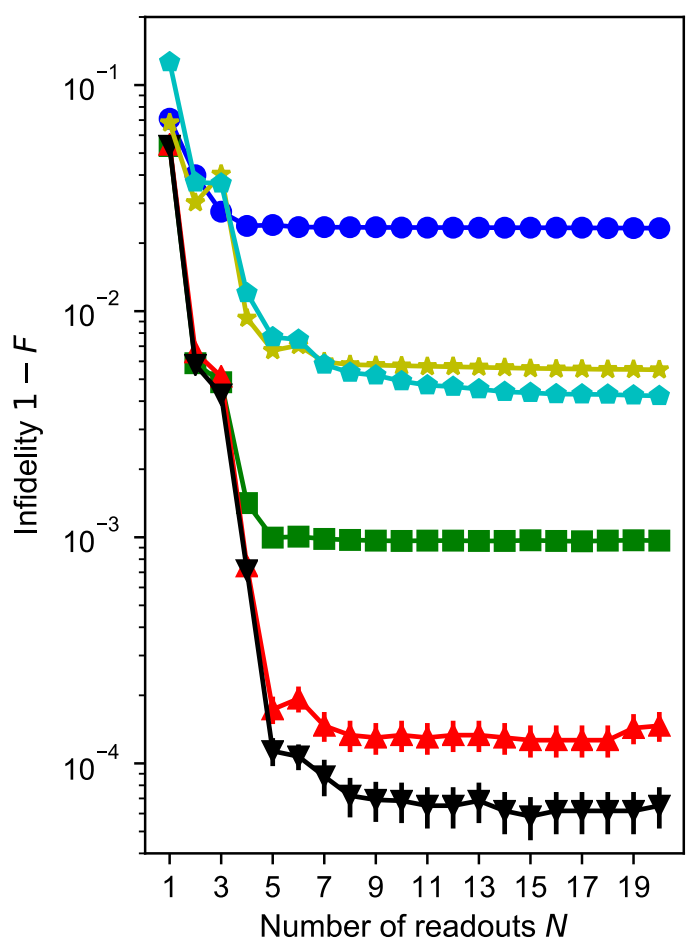

FIG. 6. High-fidelity measurement of bosonic codes. Results of measuring the logical $Z$ observable in different qubit encodings. Given single-shot ancilla assignments, a Bayesian classifier outputs either " $0_{L}$ " or " $1_{L}$," according to its best estimate of the initial state. As more readouts are incorporated into the estimate, the measurement infidelity decreases monotonically. Results for the $0-2,0-3,0-4$, and $0-5$ Fock codes are shown in blue circles, green squares, red triangles, and black triangles, respectively. Results for the first- and second-order binomial codes are shown in yellow stars and cyan pentagons, respectively. As the code distance increases, the measurement fidelity improves.

events appear to be due to rare excitations to high levels in the ancilla, the origin of which is unclear [48]. It is worth pointing out that we do this postselection only in order to compare to the theory, not to make fair qubit measurements. We emphasize that the results in the remainder of this paper, in particular, Fig. 6 and Table I, are not postselected in this way. They represent "fair" measurements.

In addition to the Fock-based codes described above, certain QEC codes can also be measured using the same procedure. The only difference in the implementation of the protocol is the choice of $S$, which is summarized in Table I. In addition to the Fock-based codes, we also study two binomial codes [25]. Such codes are based on superpositions of photon number states and are designed to correct for different combinations of photon loss, photon gain, and dephasing errors. A universal gate set and error correction have been implemented for the lowest-order binomial code [49], and these codes find application in quantum metrology [50]. In this work, we do not consider codes in which any number state appears 
TABLE I. List of codes, mapping operations, and results. Here, we list the bosonic codes studied, along with their logical $Z$ codewords, the distance between codewords with respect to the lowering operator $a$, and the subset of photon numbers for which the ancilla is excited. Experimental measurement infidelities are listed according to the logical encoding and quoted for the number $N$ of measurements which minimizes each. The best value for each class of code is highlighted in boldface.

\begin{tabular}{lccccc}
\hline \hline Code & Flip ancilla if $n \in$ & $\left|0_{L}\right\rangle$ & $\left|1_{L}\right\rangle$ & Distance & Measurement infidelity \\
\hline Fock & $\{0,1\}$ & $|0\rangle$ & $|2\rangle$ & 2 & $(2.33 \pm 0.03) \times 10^{-2}$ \\
& & $|3\rangle$ & 3 & $(9.6 \pm 0.6) \times 10^{-4}$ \\
& & $|4\rangle$ & 4 & $(1.3 \pm 0.2) \times 10^{-4}$ \\
& & $|5\rangle$ & 5 & $(\mathbf{5 . 8} \pm \mathbf{1 . 3}) \times \mathbf{1 0}^{-5}$ \\
Binomial $(\hat{s})$ & $\{1,2\}$ & $|2\rangle$ & $(|0\rangle+|4\rangle) / \sqrt{2}$ & 2 & $(5.5 \pm 0.2) \times 10^{-3}$ \\
Binomial $\left(\hat{s}, \hat{s}^{\dagger}\right)$ & $\{1,2,3\}$ & $|3\rangle$ & $(|0\rangle+|6\rangle) / \sqrt{2}$ & 3 & $(\mathbf{4 . 2} \pm \mathbf{0 . 2}) \times \mathbf{1 0}^{-3}$ \\
\hline \hline
\end{tabular}

in both the $\left|0_{L}\right\rangle$ and $\left|1_{L}\right\rangle$ states, which means that the POVM of Eqs. (1) and (2) does not depend on the phase of the number states $S$ studied. It is, therefore, sufficient to prepare number states as inputs to the measurement protocol and average the results appropriately.

Although majority voting is a convenient way to make assignments given a measurement record, it is not optimal. In particular, the measurement fidelity worsens when too many readouts are incorporated into the majority vote because of state relaxation. To make a uniform comparison between different codes, we use a Bayesian classifier [maximum likelihood estimator (MLE)]. Such a classification scheme is optimal and sufficiently general to classify any code [26]. The results of such a classification scheme are shown in Fig. 6 for Fock and binomial codes, as a function of the number of readouts taken. As more readouts are included in the classification, the infidelity improves monotonically, as expected for an MLE. Furthermore, the minimum infidelity improves as the distance of the encoding is increased. The final results are compiled in Table I, along with the definitions of $\left|0 / 1_{L}\right\rangle$ and $S$. We note, in particular, that we achieve a measurement fidelity of $1-6.5 \times 10^{-5} \approx 0.9999$ when discriminating between states $|0\rangle$ and $|5\rangle$, and $1-4.2 \times 10^{-3} \approx 0.996$ when discriminating between states $\left|0_{L}\right\rangle$ and $\left|1_{L}\right\rangle$ in the $S=2, N=1$ binomial code, both of which surpass the highest measurement fidelities reported in a circuit QED system [22].

We expect that one could measure a binomial code with higher fidelity by selecting a higher-order code or by taking more care to design a mapping pulse with a small error (e.g., by using optimal control algorithms [51-53]). However, the exact improvement depends on several factors, including the choice of $S$, which correctly balances the contributions of photon loss and gain for each code. Furthermore, improvements in cavity operations may enable future shelving schemes at the logical qubit level. For example, before measuring a binomial code, one could first apply a unitary operation which translates the code into a Fock-based encoding, in order to leverage the high measurement fidelity of that code. Such experiments would be analogous to the shelving demonstration of Sec. III.

\section{CONCLUSIONS}

We have shown that multilevel encodings can be leveraged to improve measurement fidelity in quantum information systems. This idea was explored in two contexts. In the first set of experiments, the multiply excited states of a transmon qubit were used to protect measurements against errors due to state relaxation. Furthermore, it was shown how this technique can be used to mitigate SPAM errors. In the second set of experiments, a repeated readout scheme was used to measure qubits encoded in a harmonic storage mode. In this scheme, repeated readouts suppress the effect of mapping and readout errors, and the distance between codewords suppresses the effect of state relaxation. Using an adaptive reset scheme relying on real-time feedforward logic, this scheme was implemented with a single ancilla.

Measurement is a crucial resource in quantum information processing. In particular, reliable measurements are necessary for high-fidelity teleported operations [11], which are an important component of a modular architecture for quantum computation [54]. Therefore, we expect our results to find applications in a variety of future experiments.

\section{ACKNOWLEDGMENTS}

The authors thank C. J. Axline for assistance with sample preparation during an early stage of this experiment, N. Frattini and K. Sliwa for providing the Josephson parametric converter, and N. Ofek for providing the logic for the FPGA control system used in this experiment. Facilities use was supported by the Yale SEAS clean room and Yale Institute for Nanoscience and Quantum Engineering (YINQE). This research was supported by the U.S. Army Research Office Grants No. W911NF-18-10212 and No. W911NF-16-1-0349 and by the AFOSR MURI (FA9550-14-1-0052). L. J. acknowledges support from the Packard Foundation (2013-39273). C. T. H. acknowledges support from the NSF GRFP (DGE1752134). 
[1] C. Monroe, D. M. Meekhof, B. E. King, W. M. Itano, and D. J. Wineland, Demonstration of a Fundamental Quantum Logic Gate, Phys. Rev. Lett. 75, 4714 (1995).

[2] P. W. Shor, Scheme for Reducing Decoherence in Quantum Computer Memory, Phys. Rev. A 52, R2493 (1995).

[3] E. Knill and R. Laflamme, Theory of Quantum ErrorCorrecting Codes, Phys. Rev. A 55, 900 (1997).

[4] J. Chiaverini, D. Leibfried, T. Schaetz, M. D. Barrett, R. B. Blakestad, J. Britton, W. M. Itano, J. D. Jost, E. Knill, C. Langer, R. Ozeri, and D. J. Wineland, Realization of Quantum Error Correction, Nature (London) 432, 602 (2004).

[5] N. Ofek, A. Petrenko, R. Heeres, P. Reinhold, Z. Leghtas, B. Vlastakis, Y. Liu, L. Frunzio, S. M. Girvin, L. Jiang, M. Mirrahimi, M. H. Devoret, and R. J. Schoelkopf, Extending the Lifetime of a Quantum Bit with Error Correction in Superconducting Circuits, Nature (London) 536, 441 (2016).

[6] S. Rosenblum, P. Reinhold, M. Mirrahimi, L. Jiang, L. Frunzio, and R. J. Schoelkopf, Fault-Tolerant Detection of a Quantum Error, Science 361, 266 (2018).

[7] C. H. Bennett and G. Brassard, Quantum Cryptography: Public Key Distribution and Coin Tossing, in Proceedings of the IEEE International Conference on Computers, Systems and Signal Processing (IEEE, New York, 1984), Vol. 175.

[8] V. Giovannetti, S. Lloyd, and L. Maccone, QuantumEnhanced Measurements: Beating the Standard Quantum Limit, Science 306, 1330 (2004).

[9] C. H. Bennett, G. Brassard, C. Crépeau, R. Jozsa, A. Peres, and . K. Wootters, Teleporting an Unknown Quantum State via Dual Classical and Einstein-Podolsky-Rosen Channels, Phys. Rev. Lett. 70, 1895 (1993).

[10] M. D. Barrett, J. Chiaverini, T. Schaetz, J. Britton, W. M. Itano, J. D. Jost, E. Knill, C. Langer, D. Leibfried, R. Ozeri, and D. J. Wineland, Determinsitic Quantum Teleportation of Atomic Qubits, Nature (London) 429, 737 (2004).

[11] K. S. Chou, J. Z. Blumoff, C. S. Wang, P. C. Reinhold, C. J. Axline, Y. Y. Gao, L. Frunzio, M. H. Devoret, L. Jiang, and R. J. Schoelkopf, Deterministic Teleportation of a Quantum Gate between Two Logical Qubits, Nature (London) 561, 368 (2018).

[12] A. Aspect, P. Grangier, and G. Roger, Experimental Realization of Einstein-Podolsky-Rosen-Bohm Gedankenexperiment: A New Violation of Bell's Inequalities, Phys. Rev. Lett. 49, 91 (1982).

[13] A. Aspect, J. Dalibard, and G. Roger, Experimental Test of Bell's Inequalities Using Time-Varying Analyzers, Phys. Rev. Lett. 49, 1804 (1982).

[14] E. Knill, D. Leibfried, R. Reichle, J. Britton, R. B. Blakestad, J. D. Jost, C. Langer, R. Ozeri, S. Seidelin, and D. J. Wineland, Randomized Benchmarking of Quantum Gates, Phys. Rev. A 77, 012307 (2008).

[15] D. B. Hume, T. Rosenband, and D. J. Wineland, HighFidelity Adaptive Qubit Detection through Repetitive Quantum Nondemolition Measurements, Phys. Rev. Lett. 99, 120502 (2007).

[16] A. H. Myerson, D. J. Szwer, S. C. Webster, D. T. C. Allcock, M. J. Curtis, G. Imreh, J. A. Sherman, D. N. Stacey, A. M. Steane, and D. M. Lucas, High-Fidelity Readout of
Trapped-Ion Qubits, Phys. Rev. Lett. 100, 200502 (2008).

[17] A. Seif, K. A. Landsman, N. M. Linke, C. Figgatt, C. Monroe, and M. Hafezi, Machine Learning Assisted Readout of Trapped-Ion Qubits, J. Phys. B 51, 174006 (2018).

[18] P. Harvey-Collard, B. D’Anjou, M. Rudolph, N. T. Jacobson, J. Dominguez, G. A. Ten Eyck, J. R. Wendt, T. Pluym, M. P. Lilly, W. A. Coish, M. Pioro-Ladrière, and M. S. Carroll, High-Fidelity Single-Shot Readout for a Spin Qubit via an Enhanced Latching Mechanism, Phys. Rev. X 8, 021046 (2018).

[19] A. Morello, J. J. Pla, F. A. Zwanenburg, K. W. Chan, K. Y. Tan, H. Huebl, M. Möttönen, C. D. Nugroho, C. Yang, J. A. van Donkelaar, A. D. C. Alves, D. N. Jamieson, C. C. Escott, L. C. L. Hollenberg, R. G. Clark, and A. S. Dzurak, Single-Shot Readout of an Electron Spin in Silicon, Nature (London) 467, 687 (2010).

[20] R. Hanson, L. P. Kouwenhoven, J. R. Petta, S. Tarucha, and L. M. K. Vandersypen, Spins in Few-Electron Quantum Dots, Rev. Mod. Phys. 79, 1217 (2007).

[21] A. Wallraff, D. I. Schuster, A. Blais, L. Frunzio, J. Majer, M.H. Devoret, S. M. Girvin, and R. J. Schoelkopf, Approaching Unit Visibility for Control of a Superconducting Qubit with Dispersive Readout, Phys. Rev. Lett. 95, 060501 (2005).

[22] T. Walter, P. Kurpiers, S. Gasparinetti, P. Magnard, A. Potočnik, Y. Salathé, M. Pechal, M. Mondal, M. Oppliger, C. Eichler, and A. Wallraff, Rapid High-Fidelity Single-Shot Dispersive Readout of Superconducting Qubits, Phys. Rev. Applied 7, 054020 (2017).

[23] J. Gambetta, W. A. Braff, A. Wallraff, S. M. Girvin, and R. J. Schoelkopf, Protocols for Optimal Readout of Qubits Using a Continuous Quantum Nondemolition Measurement, Phys. Rev. A 76, 012325 (2007).

[24] D. I. Schuster, A. A. Houck, J. A. Schreier, A. Wallraff, J. M. Gambetta, A. Blais, L. Frunzio, J. Majer, B. Johnson, M. H. Devoret, S. M. Girvin, and R. J. Schoelkopf, Resolving Photon Number States in a Superconducting Circuit, Nature (London) 445, 515 (2007).

[25] M. M. Michael, M. Silveri, R. T. Brierley, V. V. Albert, J. Salmilehto, L. Jiang, and S. M. Girvin, New Class of Quantum Error-Correcting Codes for a Bosonic Mode, Phys. Rev. X 6, 031006 (2016).

[26] C. T. Hann, S. S. Elder, C. S. Wang, K. Chou, R. J. Schoelkopf, and L. Jiang, Robust Readout of Bosonic Qubits in the Dispersive Coupling Regime, Phys. Rev. A 98, 022305 (2018).

[27] J. Koch, T. M. Yu, J. Gambetta, A. A. Houck, D. I. Schuster, J. Majer, A. Blais, M. H. Devoret, S. M. Girvin, and R. J. Schoelkopf, Charge-Insensitive Qubit Design Derived from the Cooper Pair Box, Phys. Rev. A 76, 042319 (2007).

[28] J. A. Schreier, A. A. Houck, J. Koch, D. I. Schuster, B. R. Johnson, J. M. Chow, J. M. Gambetta, J. Majer, L. Frunzio, M. H. Devoret, S. M. Girvin, and R. J. Schoelkopf, Suppressing Charge Noise Decoherence in Superconducting Charge Qubits, Phys. Rev. B 77, 180502(R) (2008).

[29] D. Gottesman, A. Kitaev, and J. Preskill, Encoding a Qubit in an Oscillator, Phys. Rev. A 64, 012310 (2001).

[30] Z. Leghtas, G. Kirchmair, B. Vlastakis, R. J. Schoelkopf, M. H. Devoret, and M. Mirrahimi, Hardware-Efficient 
Autonomous Quantum Memory Protection, Phys. Rev. Lett. 111, 120501 (2013).

[31] Victor . V. Albert, K. Noh, K. Duivenvoorden, D. J. Young, R. T. Brierley, P. Reinhold, C. Vuillot, L. Li, C. Shen, S. M. Girvin, B. M. Terhal, and L. Jiang, Performance and Structure of Single-Mode Bosonic Codes, Phys. Rev. A 97, 032346 (2018).

[32] L. Li, D. J. Young, V. V. Albert, K. Noh, C.-L. Zou, and L. Jiang, Designing Good Bosonic Quantum Codes via Creating Destructive Interference, arXiv:1901.05358.

[33] M. Y. Niu, I. L. Chuang, and J. H. Shapiro, HardwareEfficient Bosonic Quantum Error-Correcting Codes Based on Symmetry Operators, Phys. Rev. A 97, 032323 (2018).

[34] I. L. Chuang, D. W. Leung, and Y. Yamamoto, Bosonic Quantum Codes for Amplitude Damping, Phys. Rev. A 56, 1114 (1997).

[35] P. T. Cochrane, G. J. Milburn, and W. J. Munro, Macroscopically Distinct Quantum-Superposition States as a Bosonic Code for Amplitude Damping, Phys. Rev. A 59, 2631 (1999).

[36] M. A. Nielsen and I. L. Chuang, Quantum Computation and Quantum Information (Cambridge University Press, Cambridge, England, 2010).

[37] An anharmonic oscillator is not necessarily characterized by a single $T_{1}$ value [38]. In this context, the expression $\left(t_{m} / T_{1}\right)^{n}$ must be regarded as a rule of thumb and not an exact value.

[38] R. J. Schoelkopf, A. A. Clerk, S. M. Girvin, K. W. Lehnert, and M. H. Devoret, Quantum Noise in Mesoscopic Physics (Springer, New York, 2003), chapter Qubits as Spectrometers of Quantum Noise, pp. 175-203.

[39] B. D'Anjou and W. A. Coish, Enhancing Qubit Readout through Dissipative Sub-Poissonian Dynamics, Phys. Rev. A 96, 052321 (2017).

[40] This method of state preparation is analogous to the one described in Supplemental Material [41].

[41] See Supplemental Material at http://link.aps.org/ supplemental/10.1103/PhysRevX.10.011001 for additional information about system parameters, simulations, and analysis.

[42] F. Mallet, F. R. Ong, A. Palacios-Laloy, F. Nguyen, P. Bertet, D. Vion, and D. Esteve, Single-Shot Qubit Readout in Circuit Quantum Electrodynamics, Nat. Phys. 5, 791 (2009).

[43] J. M. Chow, J. M. Gambetta, L. Tornberg, Jens Koch, Lev S. Bishop, A. A. Houck, B. R. Johnson, L. Frunzio, S. M. Girvin, and R. J. Schoelkopf, Randomized Benchmarking and Process Tomography for Gate Errors in a Solid-State Qubit, Phys. Rev. Lett. 102, 090502 (2009).
[44] Z. Chen, J. Kelly, C. Quintana, R. Barends, B. Campbell, Yu Chen, B. Chiaro, A. Dunsworth, A. G. Fowler, E. Lucero, E. Jeffrey, A. Megrant, J. Mutus, M. Neeley, C. Neill, P. J. J. O’Malley, P. Roushan, D. Sank, A. Vainsencher, J. Wenner, T. C. White, A. N. Korotkov, and J. M. Martinis, Measuring and Suppressing Quantum State Leakage in a Superconducting Qubit, Phys. Rev. Lett. 116, 020501 (2016).

[45] G. Lindblad, On the Generators of Quantum Dynamical Semigroups, Commun. Math. Phys. 48, 119 (1976).

[46] M. Mirrahimi, Z. Leghtas, V. V Albert, S. Touzard, R. J. Schoelkopf, L. Jiang, and M. H. Devoret, Dynamically Protected Cat-Qubits: A New Paradigm for Universal Quantum Computation, New J. Phys. 16, 045014 (2014).

[47] M. Pechal, L. Huthmacher, C. Eichler, S. Zeytinoğlu, A. A. Abdumalikov, Jr., S. Berger, A. Wallraff, and S. Filipp, Microwave-Controlled Generation of Shaped Single Photons in Circuit Quantum Electrodynamics, Phys. Rev. X 4, 041010 (2014).

[48] See Supplemental Material [41] for a detailed discussion of ancilla reset errors.

[49] L. Hu, Y. Ma, W. Cai, X. Mu, Y. Xu, W. Wang, Y. Wu, H. Wang, Y. P. Song, C.-L. Zou, S. M. Girvin, L-M. Duan, and L. Sun, Quantum Error Correction and Universal Gate Set Operation on a Binomial Bosonic Logical Qubit, Nat. Phys. 15, 503 (2019).

[50] S. Zhou, M. Zhang, J. Preskill, and L. Jiang, Achieving the Heisenberg Limit in Quantum Metrology Using Quantum Error Correction, Nat. Commun. 9, 78 (2018).

[51] N. Khaneja, T. Reiss, C. Kehlet, T. Schulte-Herbrüggen, and S. J. Glaser, Optimal Control of Coupled Spin Dynamics: Design of NMR Pulse Sequences by Gradient Ascent Algorithms, J. Magn. Reson. 172, 296 (2005).

[52] P. de Fouquieres, S. G. Schirmer, S. J. Glaser, and I. Kuprov, Second Order Gradient Ascent Pulse Engineering, J. Magn. Reson. 212, 412 (2011).

[53] R. W. Heeres, P. Reinhold, N. Ofek, L. Frunzio, L. Jiang, M. H. Devoret, and R. J. Schoelkopf, Implementing a Universal Gate Set on a Logical Qubit Encoded in an Oscillator, Nat. Commun. 8, 94 (2017).

[54] L. Jiang, J. M. Taylor, A. S. Sørensen, and M. D. Lukin, Distributed Quantum Computation Based on Small Quantum Registers, Phys. Rev. A 76, 062323 (2007).

Correction: The footnote indicating the present address for author Liang Jiang was mistakenly associated with a different author during production and has been fixed. 